\title{
M1 and M2 polarization of macrophages: a mini-review
}

\author{
Kun Yeong Lee \\ Department Laboratory Medicine and Pathology, Mayo Clinic, Rochester, MN, USA
}

Received May 9, 2018

Revised May 28, 2018

Accepted May 30, 2018

Corresponding author

Kun Yeong Lee

Department Laboratory Medicine

and Pathology, Mayo Clinic, Stabile

2-42, 200 First St. SW, Rochester,

MN55905, USA

Tel: +1-507-538-0042

E-mail: Lee.Kun@mayo.edu

ORCID:

https://orcid.org/0000-0002-5165-2856
The tissue microenvironment regulates differentiation of precursor monocytes into macrophages. In response to the presence of microbial products, stimulated lymphocytes, or damaged cells in the microenvironment, macrophages differentiate into distinct functional populations. The M1 macrophages are characterized by the generation of high levels of pro-inflammatory cytokines, antimicrobial properties, increased production of reactive nitrogen and oxygen intermediates, and induction of Th1 response. In contrast, M2 macrophages are characterized by their involvement in tissue remodeling, immune regulation, tumor promotion, and efficient phagocytosis. In this minireview, we discuss the stimulation, markers, cytokines, and signaling molecules involved in macrophage polarization.

Keywords: Macrophages; Polarization; Tumor-associated macrophages

\section{INTRODUCTION}

Macrophages are the most diverse cells of the immune system that are critical for the maintenance of homeostasis. They engulf and digest microbial pathogens, foreign objects, dead cell debris, and cancer cells in a process called phagocytosis, and phagocytes are present in almost all tissues [1] of the human body (Table 1). Macrophages also regulate innate and adaptive immune responses by recruiting other immune cells such as lymphocytes. They tune various factors to perform these functions post-differentiation. Macrophages undergo polarization to M1 or M2 macrophages in response to environmental signals [2]. In this review, I summarize the stimulation signal, cytokine production, and signaling molecules associated with macrophage polarization.
Table 1. Anatomical location and tissue-specific names of macrophages

\begin{tabular}{ll}
\hline \multicolumn{1}{c}{ Location } & \multicolumn{1}{c}{ Cell name } \\
\hline Adipose tissue acrophages & Adipose tissue macrophages \\
Bone & Osteoclasts \\
Bone marrow/blood & Monocytes \\
Central nervous system & Microglia \\
Connective tissue & Tissue macrophages leading to giant \\
& cells \\
Granulomas & Epithelioid cells \\
Kidney & Intraglomerular mesangial cells \\
Liver & Kupffer cells \\
Lymph nodes & Sinus histiocytes \\
Peritoneal cavity & Peritoneal macrophages \\
Peyer's patch & LysoMac \\
Placenta & Hofbauer cells \\
Pulmonary alveoli of lungs & Alveolar macrophages \\
Red pulp of spleen & Red pulp macrophages \\
Skin and mucosa & Langerhans cells \\
\hline
\end{tabular}




\section{M1 MACROPHAGES}

M1-polarized macrophages, also known as classically activated macrophages, are activated by the cytokine interferon- $\gamma(\mathrm{IFN}-\gamma)$ and are involved in helper T cell (Th) 1 response to infection (Fig. 1). M1 macrophages produce pro-inflammatory molecules, including tumor necrosis factor (TNF)- $\alpha$ and interleukin (IL)-1, -6, -12, -23. M1 macrophages are critical for host protection against viruses and intracellular bacteria during acute infections or tumors. They produce microbicidal and tumoricidal reagents, such as nitric oxide (NO) or reactive oxygen intermediates (ROI) [3,4]. IFN- $\gamma$, lipopolysaccharide (LPS), and granulocyte-macrophage colony stimulation factor (GM-CSF) induce the polarization of macrophages into M1 type. Although macrophages produce IFN $-\gamma$, Th1 lymphocytes are the main source of IFN- $\gamma$ in the body. IFN- $\gamma$ is required for classical macrophage activation, and controls the expression of macrophage genes encoding cytokine receptors, cell activation markers, and cell adhesion molecules. LPS promotes classical macrophage activation via the toll-like receptor (TLR) 4. GM-CSF also promotes M1 polarization [4]. However, M1 macrophages are sometimes implicated in sustained inflammation and can be harmful for host health.

\section{M2 MACROPHAGES}

M2-polarized macrophages [5], also known as alternatively activated macrophages, are important in tissue repair, although they are also involved in chronic infectious diseases [6]. M2 macrophages were further divided into M2a, M2b, M2c, and M2d subtypes based on the applied stimuli and transcriptional changes $[7,8]$. The common characteristics of this subpopulation are high IL-10 and low IL-12 levels, and one of the signatures is generation of arginase-1 (Arg-1). High level of Arg-1 depletes L-arginine, which impairs $\mathrm{T}$ cell proliferation and IFN- $\gamma$ production. The increased level of Arg-1 further competes with iNOS for L-arginine and reduces $\mathrm{NO}$ production.

M2a polarization is stimulated by IL-4, IL-13, or fungal and helminth infections, and is related to Th2 immune response. Th2 cells, eosinophils, basophils, and macrophages produce IL- 4 and are important for parasite encapsulation. M2b macrophages are activated by immune complex plus TLR or IL-1 receptor ligands, which thereafter regulate immune responses. IL-10 production and antigen presentation is up-regulated (MHC II, CD86), whereas IL-12 production is down-regulated. M2c macrophages are stimulated by IL-10 and transforming growth factor (TGF) $-\beta$ and contribute to tissue remodeling and extracellular matrix

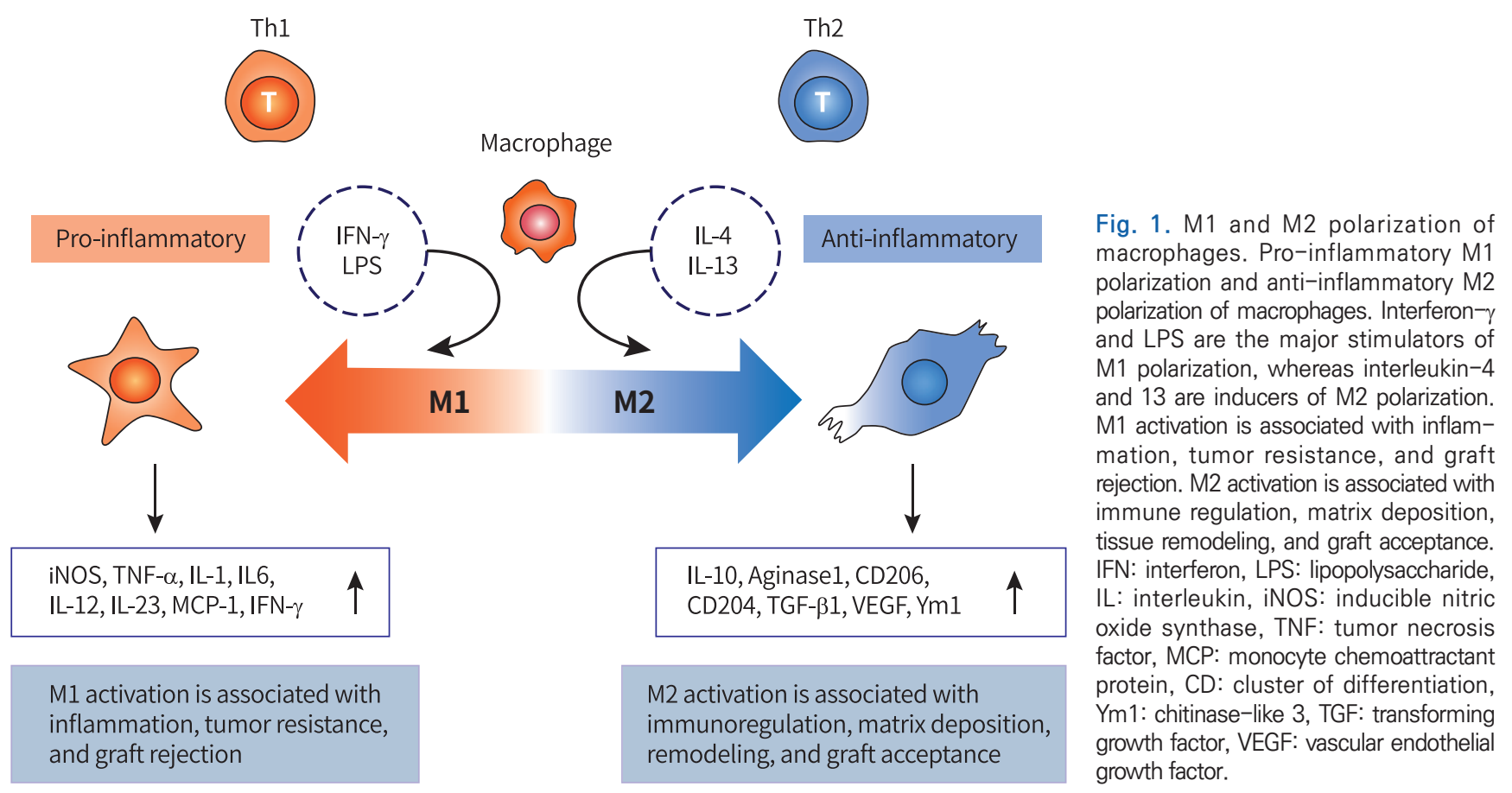


production. Glucocorticoids also influence adherence, dissemination, apoptosis, and phagocytosis of M2c macrophages [4]. Polarization into M2d is activated by IL6 and adenosine.

\section{TUMOR-ASSOCIATED MACROPHAGES}

Certain macrophages of ambiguous phenotypes, such as tumor-associated macrophages (TAM), closely resemble M2-polarized macrophages [5], are activated by tumors, and stimulate tumor cells during carcinogenesis. TAMs, as prominent immune cells in tumors, orchestrate several factors in the tumor microenvironment [9]. They play an important role in connecting inflammation with cancer (Fig. 2) by promoting proliferation, invasion, and metastasis of tumor cells, stimulating tumor angiogenesis, and inhibiting antitumor immune response mediated by T cells. The tumor-promoting role of macrophages in inflammation is supported by several lines of evidence, including genetic analysis [10,11]. Inflammatory reactions and infiltrated macrophages can promote tumor progression. This raises the possibility that the molecules/cells involved in the TAM-induced tumorigenesis might represent novel and valuable therapeutic targets for cancers [3]. TAMs produce high levels of immu-

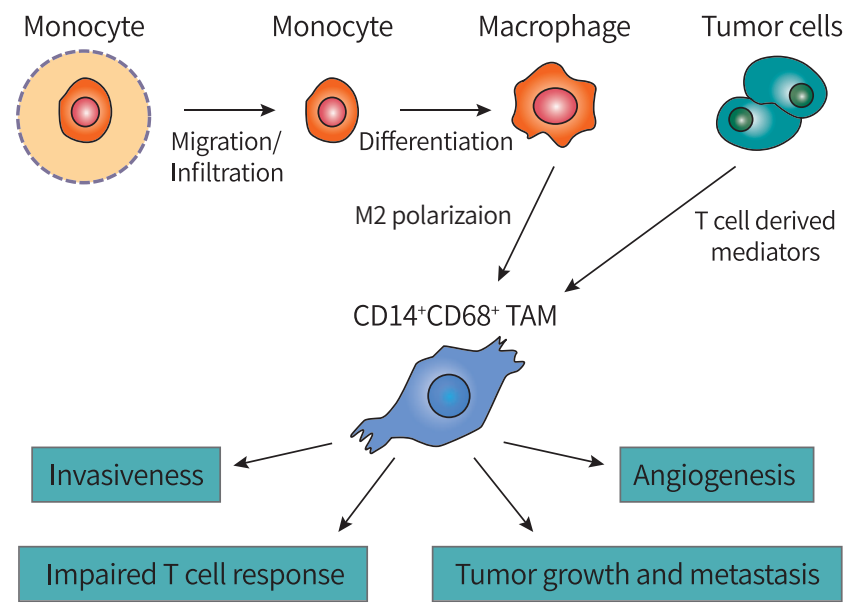

Fig. 2. Role of tumor-associated macrophages. Polarization and role of TAMs in the tumor environment. Monocytes in the bloodstream migrate into tissues, undergo polarization similar to M2 type, and become $\mathrm{CD}_{14}{ }^{+} \mathrm{CD} 68^{+} \mathrm{TAM}$. TAMs interact with tumor cells, produce cytokines, stimulate tumor growth, metastasis, invasiveness, and angiogenesis, and inhibit T cell immune response. TAM: tumor-associated macrophage.

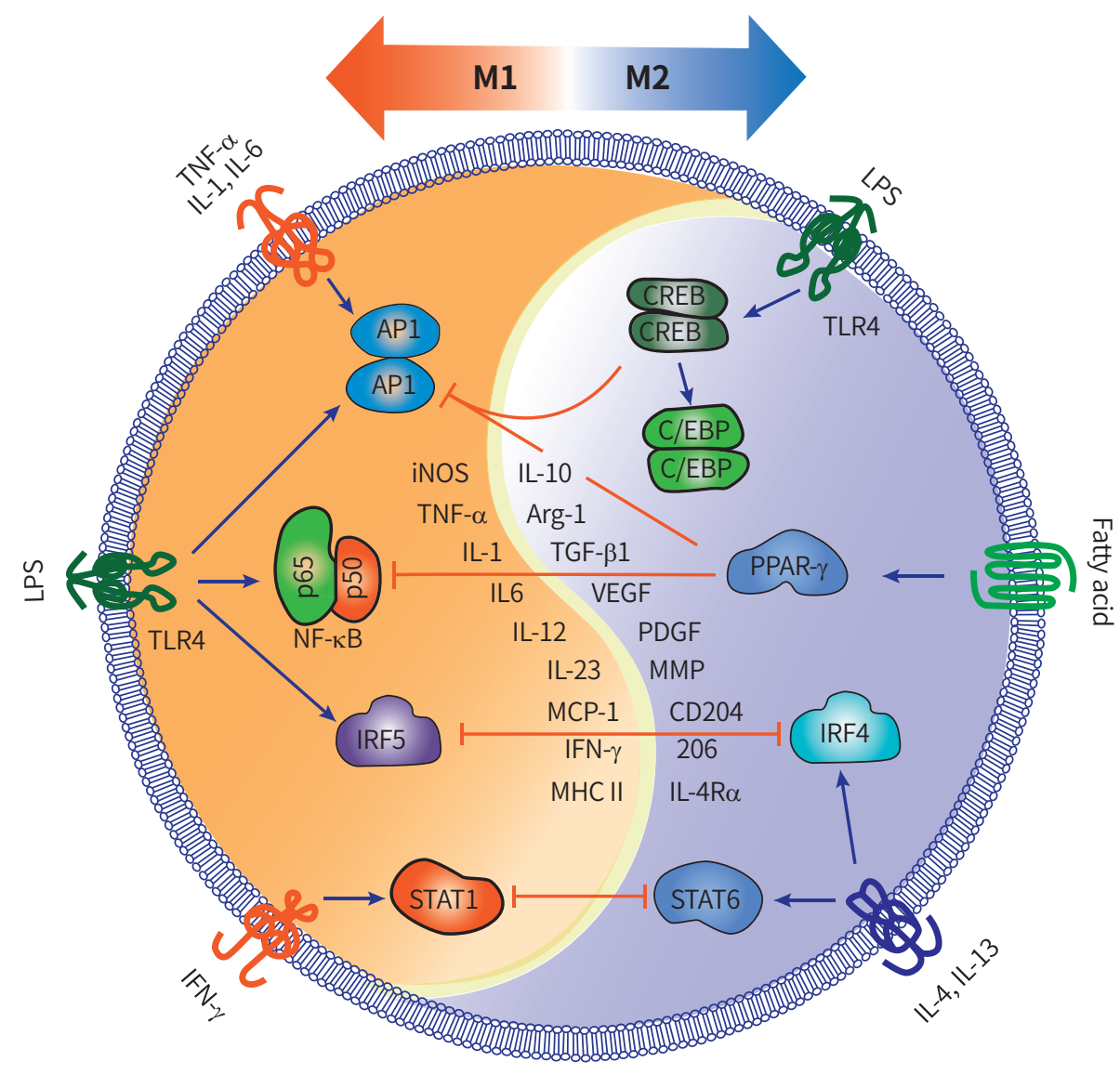

Fig. 3. Signaling pathways of macrophage polarization. Schematic showing several mechanisms of macrophage polarization and the interactive regulation of transcription factors between M1 and M2 signaling. STAT1 is activated by IFN- $\gamma$ receptor. IRF5, NF- $\kappa \mathrm{B}$, and AP1 are activated by TLR4. Enhanced AP1 expression is mediated by cytokine receptors. IL-4 receptor signaling activates STAT6 and IRF4. The fatty acid receptor activates PPAR $\gamma$. TLR4 enhances CREB and C/EBP levels. The feedback regulation between $\mathrm{M} 1$ and $\mathrm{M} 2$ are regulated by the transcription factors and cytokines. AP1: activator protein 1, NF$\kappa \mathrm{B}$ : nuclear factor of kappa light chain, IRF: interferon-regulatory factor, STAT: signal transducer and activator of transcription, CREB: cAMP-responsive element-binding protein, C/EBP: CCAAT-enhancer-binding protein, PPAR: peroxisome proliferatoractivated receptor. 
nosuppressive cytokines (IL-10 and TGF- $\beta$ ) and low levels of inflammatory cytokines (IL-12, IL-1 $\beta$, TNF $\alpha$, IL-6) [12].

\section{SIGNALING MOLECULES INVOLVED IN MACROPHAGE POLARIZATION}

The microenvironment regulates the interconversion of M1 and M2 macrophages [13]. Many key transcription factors are involved in macrophage polarization (Fig. 3). M1 macrophages express transcription factors such as nuclear factor of kappa light chain gene enhancer (NF- $\left.{ }^{\kappa} \mathrm{B}\right)$, activator protein 1 (AP-1), signal transducer and activator of transcription (STAT) 1, and interferon-regulatory factor (IRF)-5 [14-16. M2-polarized macrophages express STAT6, IRF-4, peroxisome proliferator-activated receptor (PPAR) $-\gamma$, and cAMP-responsive element-binding protein (CREB) [17-20]. These transcription factors cross-interact, express several cytokines, and regulate the conversion of macrophages to certain phenotypes (Fig. 3). The balance of these factors in pro- or anti-inflammatory functions is critical for host defense, whereas their dissonance might be detrimental for the body.

\section{CONCLUSIONS}

In this review, I summarized current information on macrophage polarization, function, and signaling. Macrophages play important roles in host defense against microbial infections and tumors, and in tissue repair. These pivotal roles are inevitably implicated in diseases such as inflammation and cancer. A better understanding of macrophage polarization is critical for their clinical application for improving the body's immune defense. Targeting polarization into TAMs is a promising strategy for cancer treatment.

\section{CONFLICT OF INTEREST}

No potential conflict of interest relevant to this article was reported.

\section{REFERENCES}

1. Ovchinnikov DA. Macrophages in the embryo and beyond: much more than just giant phagocytes. Genesis 2008;46:44762.

2. Mantovani A, Sozzani S, Locati M, Allavena P, Sica A. Macrophage polarization: tumor-associated macrophages as a paradigm for polarized M2 mononuclear phagocytes. Trends Immunol 2002;23:549-55.

3. Sica A, Larghi P, Mancino A, Rubino L, Porta C, Totaro MG, et al. Macrophage polarization in tumour progression. Semin Cancer Biol 2008;18:349-55.

4. Martinez FO, Gordon S. The M1 and M2 paradigm of macrophage activation: time for reassessment. F1000Prime Rep 2014;6:13.

5. Grivennikov SI, Greten FR, Karin M. Immunity, inflammation, and cancer. Cell 2010;140:883-99.

6. Benoit M, Desnues B, Mege JL. Macrophage polarization in bacterial infections. J Immunol 2008;181:3733-9.

7. Mantovani A, Sica A, Sozzani S, Allavena P, Vecchi A, Locati $M$. The chemokine system in diverse forms of macrophage activation and polarization. Trends Immunol 2004;25:67786.

8. Rőszer T. Understanding the mysterious M2 macrophage through activation markers and effector mechanisms. Mediators Inflamm 2015;2015:816460.

9. Chen Y, Zhang S, Wang Q, Zhang X. Tumor-recruited M2 macrophages promote gastric and breast cancer metastasis via M2 macrophage-secreted CHI3L1 protein. J Hematol Oncol 2017;10:36.

10. Coussens LM, Werb Z. Inflammatory cells and cancer: think different! J Exp Med 2001;193:F23-6.

11. Balkwill F, Mantovani A. Inflammation and cancer: back to Virchow? Lancet 2001;357:539-45.

12. Sica A, Saccani A, Bottazzi B, Polentarutti N, Vecchi A, van Damme J, et al. Autocrine production of IL-10 mediates defective IL-12 production and NF-kappa B activation in tumor-associated macrophages. J Immunol 2000;164:762-7.

13. Wynn TA, Chawla A, Pollard JW. Macrophage biology in development, homeostasis and disease. Nature 2013;496:44555.

14. Oeckinghaus A, Hayden MS, Ghosh S. Crosstalk in NF- $\kappa B$ signaling pathways. Nat Immunol 2011;12:695-708.

15. Schonthaler HB, Guinea-Viniegra J, Wagner EF. Targeting inflammation by modulating the Jun/AP-1 pathway. Ann Rheum Dis 2011;70 Suppl 1:i109-12.

16. Krausgruber T, Blazek K, Smallie T, Alzabin S, Lockstone H, Sahgal N, et al. IRF5 promotes inflammatory macrophage polarization and TH1-TH17 responses. Nat Immunol 2011; 12:231-8.

17. Ohmori Y, Hamilton TA. IL-4-induced STAT6 suppresses IFN-gamma-stimulated STAT1-dependent transcription in mouse macrophages. J Immunol 1997;159:5474-82. 
18. Satoh T, Takeuchi O, Vandenbon A, Yasuda K, Tanaka Y, Kumagai Y, et al. The Jmjd3-Irf4 axis regulates M2 macrophage polarization and host responses against helminth infection. Nat Immunol 2010;11:936-44.

19. Odegaard JI, Ricardo-Gonzalez RR, Goforth MH, Morel CR, Subramanian V, Mukundan L, et al. Macrophage-specific
PPARgamma controls alternative activation and improves insulin resistance. Nature 2007;447:1116-20.

20. Ruffell D, Mourkioti F, Gambardella A, Kirstetter P, Lopez RG, Rosenthal N, et al. A CREB-C/EBPbeta cascade induces M2 macrophage-specific gene expression and promotes muscle injury repair. Proc Natl Acad Sci U S A 2009;106:17475-80. 